\title{
Habiter les interstices et leurs possibilités : les discours utopiques et méta-utopiques dans Les Furtifs, «C@PTCH@ » et « Hyphe....?» d'Alain Damasio
}

\section{Introduction ${ }^{1}$}

Habiter est la première capacité des vivants, affirme Lorca, le protagoniste du roman Les Furtifs (Damasio, 2019). Elle figure au cœur de l'œuvre de l'écrivain Alain Damasio, comme en témoignent La Zone du Dehors (Damasio, 2013 [1999]) et sa société dystopique. Marquée par l'obsession sécuritaire et un système d' " assignation à personnalité [...] qui nous hiérarchise tous et qui nous attribue place, case, et rang » (p. 10), convoquant intertextuellement les classiques de la " littérature surveillancielle " (Aïm, 2020) que sont Nous autres, Le Meilleur des mondes et 1984, cette société voit s'organiser en son sein un mouvement de résistance irrépressible conduisant à l'instauration, hors de ses murs, de cités libertaires mues par le besoin d'habiter le monde autrement. En témoigne également La Horde du Contrevent (Damasio, 2004), épopée contant les hauts faits d'une poignée de nomades bravant les milieux les plus hostiles à la recherche d'espaces habitables. Au regard de la centralité de l'acte d'habiter, il serait possible de qualifier les œuvres de Damasio de « fictions écouménales ».

Christophe Duret - stagiaire postdoctoral au Laboratoire universitaire de documentation et d'observation vidéoludique de l'Université de Montréal. Adresse de correspondance : 124, rue de la Croix, J1C 0R7, Sherbrooke, Canada ; e-mail : christophe.duret@usherbrooke.ca

ORCID iD : https://orcid.org/0000-0002-2570-6849

1. Les recherches dont rend compte cet article ont été effectuées grâce au soutien financier du Fonds de recherche du Québec - Société et culture. 
L'écoumène, c'est le monde habité, soit l'ensemble des milieux humains vus comme le fruit des relations techniques et symboliques qu'une société entretient avec son environnement (Berque, 2000). Mais l'habiter désigne aussi une appropriation de l'espace (voir, notamment, Lazzarotti, 2006 ; Stock, 2004), des manières d'être aux lieux (Fries-Paiola et De Gasperin, 2014) et, donc, des pratiques habitantes (Rosselin, 2002). L'habiter, enfin, est un co-habiter ; il porte sur la dimension spatiale de la socialité, ou encore sur les relations de coexistence des individus au sein de l'espace social (Duret, 2019).

Jenkins (2006) a montré que les fictions de la culture de genres contemporaine (qui inclut la fantasy et la science-fiction, par exemple) relèvent moins de l'art de la mise en récit que de "l'art de la création de mondes » (p. 114), comme en témoignent, par exemple, des franchises telles que The Matrix, Star Wars, Terminator et The Walking Dead. La même chose pourrait être dite de l'utopie, puisqu'il y est question, selon Ruyer (1950), de " créer un monde en miniature, mais complet » (p. 123). La propension à la complétude dans l'érection d'un monde diégétique figure au cœur des « fictions écouménales " (Duret, 2019). Ces dernières représentent en effet des mondes détaillés, constituant l'objet central du récit, au sein desquels s'interpénètrent les réalités chimico-physiques (l'environnement), les réalités organiques (la biosphère) et, surtout, les réalités écouménales, soit les relations constitutives des milieux humains; le monde en tant qu'il est habité et co-habité et, par le fait même, investi par nos subjectivités, animé par nos interactions sociales et travaillé par nos techniques.

Dans les fictions écouménales de Damasio, l'habiter est foncièrement politique et figure au cœur d'une rhétorique spatiale opposant un état de société dysphorique, marqué par la dérive sécuritaire au sein de villes privatisées et surveillées, émaillées de technologies de divertissement plongeant les usagers privilégiés dans le confort, l'hébétude et l'hyperindividualisme ${ }^{2}$, d'une part, et un habiter alternatif, d'autre part, situé dans les marges et interstices des dystopies dépeintes, présent sous la forme d'un horizon d'espoir accordant une place de choix à la mixité sociale et au vivre-ensemble dans un monde aspirant à se libérer de la ségrégation socio-spatiale.

Dans cet article, nous analyserons le discours consacré par le roman Les Furtifs et les nouvelles «C@PTCH@ » et "Hyphe...? », de Damasio, à la question de l'habiter, un discours partagé entre l'eutopie (ou utopie positive) et la dystopie (ou utopie négative). Nous verrons que ces œuvres, caractéristiques de la dystopie critique, ne se contentent pas de porter une appréciation négative de la conjoncture actuelle en la matière ni de proposer une vision alternative de ce qu'habiter le monde doit ou pourrait - signifier, mais qu'elles interrogent également les limites de l'utopie classique pensée en tant que programme, ceci afin de proposer, par le biais d'un discours méta-utopique (ou discours sur le discours utopique), cette fois, une conception de l'utopie comme aspiration et comme " jeu sur les possibles latéraux à la réalité » (Ruyer, 1950, p. 9).

2. Pour une analyse du caractère dystopique de l'habiter chez Damasio, voir Duret (2021). 


\section{Utopie, dystopie et discours méta-utopique}

Avant toute chose, il convient de s'arrêter sur le terme "utopie ». Forgé par Thomas

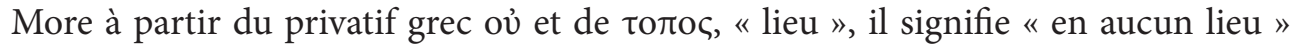
et désigne alors l'île imaginaire d'Utopia, dont la société idéale réalise le bonheur de chacun. Comme le souligne Funcke (1988), son étymologie adéquate aurait dû être "a-topia» et non "ou-topia», mais le second terme permettait de jouer sur l'ambiguiité des mots outopia et eutopia (créé à partir de عũ̃, «bon »), entre un lieu qui n'existe en aucun lieu et le bon lieu, «ou » et «eu » étant homophones pour un locuteur anglais. Outopia est axiologiquement neutre. Il désigne un lieu sans le qualifier de souhaitable ou de bon, n'appelant pas à une meilleure condition, mais à une condition autre, ce qui permet de définir ensuite deux sous-catégories de l'utopie qui, elles, sont fortement marquées, axiologiquement : l'eutopie (l'utopie positive) et son opposée, la dystopie (l'utopie négative). L’eutopie est alors définie comme « une société imaginaire décrite de manière détaillée et dont la location spatiale et temporelle suggère au lecteur contemporain qu'elle est considérablement meilleure que la société dans laquelle il vit »(Sargent, 2005, p. 9). C’est donc en tant que classe subsumant l'eutopie et la dystopie que nous entendrons le terme "utopie».

La dystopie, quant à elle, désigne "une société imaginaire décrite de manière détaillée et dont la location spatiale et temporelle suggère au lecteur contemporain qu'elle est considérablement pire que la société dans laquelle il vit » (Sargent, 2005, p. 9). Selon Booker et Thomas (2009), elle s'avère fortement satirique et sa fonction est de nous avertir des conséquences possiblement désastreuses de tendances existant déjà dans la conjoncture présente de l'œuvre (son contexte sociohistorique).

Booker et Thomas (2009) font de l'anti-utopie ou de la contre-utopie un synonyme de la dystopie, car cette dernière serait, selon eux, fréquemment conçue dans le but de critiquer les implications potentiellement néfastes de certaines formes que prend la pensée eutopique. Toutefois, en télescopant les deux concepts, on fait fi de leur étymologie respective. Les préfixes « anti- » et « contre- » expriment l'idée d'opposition. L'anti-utopie (pensons au Gulliver de Jonathan Swift, par exemple) s'oppose à la prétention totalisante de l'eutopie, présente dans ses propositions, ses projets, ses plans ou son programme. Si, comme l'écrit Munier (2013),

toute [e]utopie propose l'amendement de la société qui lui est contemporaine par la construction idéelle d'un modèle meilleur et parallèle, la contre-utopie déclare d'emblée l'inanité d'un tel projet [...] [et] l'auteur d'une contre-utopie emprunte le procédé de l'[e] utopie - la fiction d'une cité parfaite - pour la ridiculiser ». (p. 118-119)

Anti-utopie et dystopie revêtent toutes deux une dimension critique. Toutefois, dans le premier cas, l'objet de cette critique est l'eutopie, alors que dans le second, il s'agit de la société dans laquelle l’ouvre a été produite - sa conjoncture -, qu'elle considère comme hautement faillible et potentiellement néfaste si jamais ses défauts 
devaient se voir exacerbés dans le futur. Il serait donc plus juste de distinguer deux types de discours pour comprendre ce qu'est l'anti-utopie : le discours utopique (eutopique et/ou dystopique) à proprement parler et le discours sur le discours utopique, soit le discours méta-utopique (Duret, 2019). Une œuvre anti-utopique se voit donc porteuse d'un discours méta-utopique dans la mesure où elle s'exprime sur les limites (voire la dangerosité) des eutopies.

La confusion de Booker et Thomas (2009) peut s'expliquer par le fait que plusieurs classiques de la dystopie dénoncent les totalitarismes de leur époque, à l'instar du 1984 de George Orwell, dirigé explicitement contre le régime stalinien. Or, l'accusation anti-utopique selon laquelle l'eutopie est à la source des totalitarismes $\mathrm{du} \mathrm{XX}^{\mathrm{e}}$ siècle est récurrente et la dénonciation des totalitarismes ne tombe jamais bien loin de la dénonciation de l'inanité de tout projet eutopique. Comme le souligne Engélibert (2015), en prenant pour exemple les propos de Lapouge (1973), on met en opposition l'égalité de l'eutopie et la liberté de l'histoire, de sorte que la première, "sans doute parée des meilleures intentions du monde, débouche sur son contraire. Elle croit vouloir la liberté, mais transforme la cité en fourmilière et les hommes en insectes » (Engélibert, 2015, p. 242). Toujours selon Engélibert, d'autres auteurs appartenant à une certaine "vulgate conservatrice » (p. 243) - à l'instar de Faye (1993) - assimilent l'eutopie au totalitarisme. Toutefois, comme l'affirme Levitas (dans Levitas et Sargisson, 2003), tous les mouvements politiques revêtent une dimension eutopique, puisqu'ils proposent des perspectives sur ce qu'une bonne société devrait être. Si certains de ces mouvements conduisent au totalitarisme, ce n'est pas le cas de tous ; une organisation systématique et totalisante du social par le biais d'un projet politique unifié se situe à l'exact opposé, par exemple, de ce que prônent le libertarianisme, l'anarchisme ou l'objectivisme randien. Ainsi, écrit Levitas, "le problème du totalitarisme n'est pas son [e]utopisme, mais son totalitarisme » (p. 26).

Loin de cette mise en équivalence problématique de l'eutopie et du totalitarisme figurent l'eutopie critique et la dystopie critique. La première expression vient de Moylan (2014) et désigne un ensemble de fictions (Ecotopia, The Dispossessed, Trouble on Triton...) parues dans les années 1960-1970 et caractérisées par une conscience des limites de la tradition eutopique. Elles rejettent l'eutopie comme plan tout en la conservant comme rêve ou idéal - comme aspiration - et acceptent les différences et les imperfections à l'intérieur de leurs limites. Les eutopies critiques se présentent donc moins comme des systèmes clos, statiques, complets, uniformes et parfaits que comme une attitude autoréflexive et pluraliste, ne proposant jamais autre chose que des constructions partielles et faillibles (Moylan, 2000, 2014). Ici aussi, un discours méta-utopique est en jeu, dans la mesure où l'on met en exergue les limites des eutopies classiques à prétention totalisante sans pour autant renoncer à proposer en contrepartie un projet ou un processus eutopique.

Quant à la dystopie critique, elle apparaît à la fin des années 1980 avec des œuvres telles que Xenogenesis, Parable of the Sower, He, She and It et The Handmaid's Tale. Elle opère une critique de la conjoncture présente, comme le fait la dystopie classique, 
mais elle s'en distingue lorsqu'elle esquisse dans ses limites une alternative eutopique, alors que la dystopie classique maintient l'espoir en dehors de ses pages et nous avertit " que nous seuls, en tant que lecteurs, pouvons espérer échapper à son futur pessimiste " (Baccolini et Moylan, 2003, p. 7). La dystopie critique se charge d'aspirations eutopiques en réponse à « la dévaluation de l'[e]utopie par un discours néolibéral officiel qui proclame la fin de l'histoire et célèbre simultanément la fin du rêve social radical et l'achèvement d'une "[e]utopie" instantanée du marché " (Baccolini et Moylan, 2003, p. 6-7) et relève donc du discours utopique, mais comprend également une dimension méta-utopique, car elle

négocie le nécessaire pessimisme de la dystopie générique à l'aide d'une position [e]utopique ouverte et militante qui non seulement enfonce la clôture hégémonique du monde alternatif du texte, mais qui, également, refuse de manière autoréflexive la tentation anti-utopique qui se larve comme un virus latent dans tout compte-rendu dystopique. (Moylan, 2000, p. 195, nous soulignons)

Dès lors, les auteurs s'inscrivant dans ce courant

confrontent le musèlement et la cooptation simultanés de l'[e]utopie durant cette décennie en retournant les stratégies dystopiques de manière à confronter la réalité sociale en changement. Le[ur]s œuvres [...] réemploient la dystopie comme une forme narrative critique travaillant à contre-courant d'un climat économique, politique et culturel sinistre. (Baccolini et Moylan, 2003, p. 3)

Contrairement à l'eutopie critique, la dystopie critique ne propose pas un discours sur les limites de l'eutopie classique, mais elle conteste plutôt le caractère anti-utopique de la dystopie classique.

Suivant ces distinctions, les œuvres que nous examinerons sont à considérer tout à la fois comme des eutopies et des dystopies critiques, en ce sens qu'elles problématisent l'eutopie et la dystopie classiques par le biais d'un discours méta-utopique, et ce sans renoncer à dépeindre dans leurs pages un horizon d'espoir.

\section{Les dystopies de Damasio}

On doit à l'écrivain de science-fiction et de fantasy français Alain Damasio (1969-), entre autres œuvres, les romans La Zone du dehors, La Horde du Contrevent et Les Furtifs, la novella Scarlett et Novak et le recueil de nouvelles Aucun souvenir assez solide, parus entre 1999 et 2021.

En nous appuyant sur le roman Les Furtifs et les nouvelles "C@PTCH@ » et «Hyphe...? », nous examinerons la filiation de l'œuvre damasienne avec les eutopies et dystopies critiques. 
La nouvelle «C@PTCH@»(Damasio, 2012) dépeint un monde dans lequel les enfants naissent séparés de leurs parents et mènent une existence précaire en attendant de les rejoindre. Pour ce faire, ils doivent, lors de championnats appelés "c@ptch@ ", traverser la "Ville », lieu inhabité et quintessence de la surveillance numérique, quadrillé qu'il est de drones, de capteurs de mouvements et de dispositifs d'analyse biométrique. Pour remporter le championnat et retrouver leurs parents, les enfants doivent éviter les dispositifs de surveillance qui, le cas échéant, les dématérialisent. Les candidats malheureux survivent alors sous la forme de données dans le "réseau ", condamnés à une existence numérique fortement appauvrie.

La nouvelle "Hyphe...? " (Damasio, 2018), quant à elle, se déroule en 2045 dans la $\mathrm{ZAD}^{3}$ de Notre-Dame-des-Landes, alors que les communes françaises en faillite budgétaire sont vendues une à une à de grands conglomérats et deviennent des « villes libérées ». La communauté autogérée se présente comme une enclave eutopique axée sur la mise en commun et la solidarité sociale au sein d'une société néolibérale reposant sur une privatisation tous azimuts.

Les Furtifs, enfin, se déroule en 2041 dans une France dystopique marquée par le recul de l'État au profit de grandes entreprises qui, comme dans "Hyphe...? », ont graduellement privatisé les villes, lesquelles sont devenues dans l'exercice « intelligentes » et l'objet d'une surveillance exacerbée.

Un drame familial sert de fil conducteur au récit. Tishka, la fille des protagonistes Lorca et Sahar Varèse, a disparu. Lorca la soupçonne d'avoir rejoint les furtifs, des êtres capables de se métamorphoser à volonté en s'hybridant avec du vivant et du non-vivant. Les furtifs cohabitent avec les humains et sont passés maîtres dans l'art d'échapper à leur regard et aux dispositifs de surveillance. Leur existence est perçue comme une menace aux yeux du gouvernement et des entreprises qui gèrent les villes. Pour retrouver sa fille, Lorca intègre une unité de l'armée chargée d'étudier les furtifs. Cette quête familiale se double d'un projet eutopique, alors que des collectifs issus de mouvances libertaires reprennent les territoires privatisés pour en faire des zones autogouvernées. Cette lutte se verra fortement inspirée par les furtifs, dont l'existence est révélée au grand public alors que l'on découvre que Tishka, toujours vivante, s'est hybridée avec eux.

Dans le cadre de ces trois œuvres dystopiques, les textes de Damasio opposent des sociétés corpocratiques hautement surveillées à des microsociétés autogérées, construites dans les marges et interstices du pouvoir, qui demandent à faire tache d'huile, et ce avec la proposition de nouvelles manières de créer de l'être-ensemble et d'habiter le monde. De telles contrepropositions matérialisent un horizon d'espoir et donnent lieu à des dystopies critiques, alors qu'en des « temps un peu crépusculaires où l'on a du mal à se projeter positivement vers l'avenir ", nous dit l'auteur, elles par-

3. Une $\mathrm{ZAD}$, ou « zone à défendre », est un lieu occupé par des activistes de manière généralement illégale afin d'empêcher un grand projet d'aménagement, à l'instar de l'aéroport de Notre-Dame-desLandes, en France (Fougier, 2016). 
ticipent à «l'un des combats majeurs à mener, pour [l]es écrivains de science-fiction [...] et pour les citoyens actifs et militants que ces enjeux touchent, que de proposer un futur qui renoue avec l'horizon du désirable, un futur qui fasse envie » (Damasio et Lévy-Provençal, 2016, para. 5). Il s'agit, pour Damasio, d'écrire « de la science-fiction pour "laisser à désirer" des mondes possibles " (Damasio et Carabédian, 2016, p. 73). Il en résulte des œuvres dans lesquelles on matérialise l'appréciation négative de la conjoncture présente, dont les traits sont grossis jusqu’à la rendre insoutenable sous la forme d'un espace-temps dystopique, tout en matérialisant dans le même souffle l'ébauche d'organisations sociales alternatives (et eutopiques) répondant à l'amélioration de cette même conjoncture et de l'habiter qui la caractérise.

Tout en proposant des mondes à désirer et en offrant une critique de la conjoncture présente, les œuvres examinées mettent également en procès l'eutopie classique par le biais d'un discours méta-utopique. Il ne s'agit pas de dénoncer l'inanité de tout projet eutopique, toutefois, mais de questionner, à l'instar des eutopies critiques, ses traits jugés les plus problématiques, soit les caractéristiques que l'on associe aux eutopies classiques et à leur tendance totalisante. Ceci, tout en préférant à ces dernières l'eutopie comme aspiration et comme jeu sur les possibles latéraux à la réalité.

\section{Discours eutopiques et méta-utopiques dans l'œuvre damasienne}

Selon Pagès (2000), les eutopies littéraires classiques renferment plusieurs constantes sémantiques et formelles, au plan générique : l'insularité, l'intemporalité, l'autarcie, la réglementation et la planification urbaine. Nous examinerons ici comment les textes de Damasio les remettent tour à tour en question pour les confronter à leur propre vision de l'eutopie.

\subsection{L'insularité et l'autarcie}

En vertu du principe de l'insularité, la pensée eutopique privilégie les espaces isolés et protégés (Pagès, 2000). Dans Les Furtifs, cette caractéristique se manifeste par la figure de l'enclave sécurisée et témoigne d'une lecture dysphorique de la tendance contemporaine à la fermeture sur soi et à la privatopie.

L'habiter contemporain se voit marqué par un phénomène que McKenzie (1996) qualifie de «privatopie ». Il s'agit d'une tendance, marquée par le déclin de l'État providence et l'essor du capitalisme tardif, à la multiplication des enclaves privées au sein des villes (pensons aux quartiers résidentiels fermés) qui découlent d'une idéalisation de la vie communautaire. La privatopie entraîne la réduction de l'espace public et un transfert du pouvoir au profit de la surveillance privée. Il y a alors dissolution de la société civile au sein d'un monde prétendument mobile et sans frontières, un monde de divisions sociales inégalitaires (Hardt et Negri, 2000) marqué par le recul de la mixité sociale sous le coup d'une volonté de démarcation et de séparation 
spatiales et d'une privatisation de la vie publique, non seulement dans un esprit individualiste, mais également dans une logique de " clubisation " (Mongin, 2013) voulant que les individus se regroupent et habitent en fonction d'affinités électives telles que le statut socioéconomique.

Dans Les Furtifs, la privatopie se donne à voir dans la multiplication des conforteresses, copropriétés fermées et figures d'un habiter enclavé. Il s'agit d'une manifestation concrète du technococon, emblème, chez Damasio, d'une société où sont articulés plus nettement la fermeture à l'altérité et le désir de confort et de divertissement par une prise en charge technologique personnalisée. Dans Les Furtifs (Damasio, 2019), le technococon représente la dernière itération du " [c]ontrôle panique et raisonné de l'altérité » (p. 409) exercé de tout temps par l'humanité en vue d'atteindre la sérénité. Il s'agit de se mettre hors d'atteinte de l'autre par le truchement de la technologie et, plus précisément, à l'aide des dispositifs de la ville intelligente et de la domotique, dont l'intelligence ambiante permettrait soi-disant un «monde bienveillant, attentif à nous. Un monde [...] qui nous protège et nous choie, nous aide et corrige nos erreurs, qui nous filtre l'environnement et ses dangers [...] pour notre bien-être » (p. 277). Mais cette technologie est mortifère et dévitalisante, car elle enferme ses usagers dans une " bulle autour de [leur] solitude » (p. 277), une solitude reliée, loin de toute idée de communauté, vécue par l'absence croissante de lien social en face à face, dans une coupure d'avec l'altérité vécue au profit de relations tissées avec des objets connectés. Au sein du technococon, les communications numériques sont devenues " [1]'hyperlien des hyper-îliens » et leurs interfaces ont « fait naître l'alter ego, cette intelligence amie qui a permis de forclore l'altérité cruciale du rapport à nos pairs pour nous offrir, à la place, un miroir empathique et en réalité purement esclave de nos désirs » (p. 409). Le co-habiter y est dépeint comme étant « en morceaux, éparpillé en tessons incompatibles. Une cosmosaïque où chacun est bienheureux debout sur sa tesselle - mais qui ensemble ne forme plus aucune figure solidaire» (p. 410). En résulte un enfermement sur soi, une dislocation du vivre-ensemble, une " déshumanisation relationnelle et empathique qui confine à la misanthropie molle [...] et rend improbable la rencontre avec ce qui n'est pas nous» (p. 618).

En résumé, l'insularité des « hyper-1̂liens » aliène et entraîne solitude, division, refus de l'altérité et homogénéité sociale. Elle est foncièrement dystopique, car c'est bien une tendance de la conjoncture actuelle, jugée délétère, qui est mise en procès sous une forme exacerbée, mais elle problématise également la configuration spatiale par excellence de l'eutopie classique : l'île conçue comme une enclave isolée et protégée.

Pourtant, l'île n'est pas étrangère à l'impulsion eutopique de l'œuvre damasienne. Dans « Hyphe...? » (Damasio, 2018), la privatisation des villes a pour alternative la ZAD de Notre-Dame-des-Landes, qui essaime au point où l'Europe comprend $364 \mathrm{ZAD}$ en 2045. Sur les cartes, "ça fait comme des îles sur l'océan du capital » (para. 21). Mais ces îles, loin d'être des isolats, sont ouvertes sur le monde, et « un jour, ça pourrait bien former un continent » (para. 21). 
Dans Les Furtifs, Lorca peint le portrait d'une communauté intentionnelle vivant sur une île, le Javeau-Doux, présentée comme une « [e]utopie pratiquée» (Séguy, 1971). Mais par contraste avec une société hyperindividualiste et repliée sur elle-même, «le miracle opérait toujours sur le Javeau-Doux [...] où les liens étaient [...] délicatement tissés entre les gens, où l'on sentait une telle attention mutuelle " (Damasio, 2019, p. 179). Non seulement l'île est-elle le lieu d'une fine interconnexion entre les habitants, mais son ouverture face à l'altérité s'ouvre-t-elle sur le non-humain :

ces liens humains semblaient se prolonger hors du social, en rhizome à nos pieds ou à la façon de branches qui auraient poussé au bout de nos doigts, tendues vers... Vers... quoi ? Les animaux et les plantes, la terre retournée, le fleuve ? Plus loin ? Vers le cosmos? Ça sortait en tout cas du seulement-humain, du trop-humain, de l'hominite aiguë. (p. 179)

Sur le plan de l'intertextualité, le rhizome mentionné dans l'extrait précédent convoque non seulement Deleuze et Guattari (1980), figures tutélaires, avec Foucault, de l'œuvre de Damasio, mais « Hyphe...? ", également. Dans cette relation intertextuelle, l'île eutopique n'est plus une enclave isolée et protégée, mais un plateau au sein d'un rhizome, métaphore qui rend compte d'une forme d'organisation déhiérarchisée, sans début ni fin, au milieu et entre les choses. Le rhizome sétend horizontalement et forme non un centre, mais des plateaux, c'est-à-dire des "multiplicité[s] connectable $[\mathrm{s}]$ avec d'autres par tiges souterraines superficielles [...] région[s] continue[s] d'intensités, vibrant sur elle[s]-même[s], et qui se développe[nt] en évitant toute orientation sur un point culminant ou vers une fin extérieure» (p. 32). Le rhizome s'oppose à une autre métaphore, l'arbre, qui rend compte d'un « modèle linéaire centré, livré à la transcendance d'un pouvoir » (Nel, 1999, p. 131).

Dans « Hyphe...? », Damasio (2018) mobilise les métaphores de l'arbre et du rhizome et les place en opposition dans le cadre d'un double discours eutopique et méta-utopique. Le narrateur y assimile la ZAD et ses habitants - opposants à la privatopie grandissante - à la forêt. Pour beaucoup, affirme-t-il, la résistance est vue comme un arbre, figure de la verticalité : " Des résistances hautes, altières, des insurrections " (para. 21). Mais la forêt, comme la ZAD, selon lui, est plutôt une force horizontale, un rhizome, soit « [1]es fins filaments des hyphes, le mycélium qui les tresse, cette nappe qui fascicule furtivement sous la majesté très visible des houppes, c'est ça qui en vérité tient la forêt » (para. 21). L’arbre, comme l'île, n'est alors que le plateau visible d'un vaste rhizome courant sous la terre et favorisant les échanges au sein d'un réseau polytopique. Il est donc pleinement ouvert : « toujours au milieu, ni début ni fin» (Deleuze et Guattari, 1980, p. 11). Une ouverture dans les échanges qui s'oppose au caractère autarcique des eutopies classiques (Pagès, 2000) et qui confère une puissance de vie, définie par « le nombre de liaisons qu'un être est capable de tisser et d'entrelacer sans se porter atteinte [...] [une puissance qui est] [c]elle de l'écoute et de l'accueil, de l'hospitalité au neuf, qui surgit. C'est la capacité [...] à se tenir debout dans l'Ouvert» (Damasio, 2019, p. 620). 


\subsection{L'intemporalité et la réglementation}

À l'hyperindividualisme et à la pauvreté des relations interpersonnelles de la privatopie dépeinte dans Les Furtifs, la nouvelle « Hyphe...? » oppose l'hyphe, soit le principe sur lequel repose « la force des collectifs » à même de fonder un futur désirable. Ces liens qu'il tisse,

il ne faut pas les voir comme des cordes [...]. Ce sont plutôt des lacis d'actes, des gestes initiés puis répétés par untel, tel groupe, autrement, des règles pas très droites qui se ramifient à force de gniaque, un enchevêtrement de services rendus, de corvées joyeuses, d'entredons croisés. (Damasio, 2018, para. 21)

Dès lors, il n'est pas question d'établir « un système donné "une fois pour toute" [...] [un] système homogène et antidialectique ", immuable, suivant le principe de l'eutopie classique qu'est l'intemporalité (Pagès, 2000), mais plutôt un processus mouvant d'actes, « des entrelacs organiques de réunions à n'en plus finir, de solidarités d'un jour, de complicités de fond et de conflits au final dépassés » (Damasio, 2018, para. 21). Car ce sont ces entrelacs qui confèrent

la puissance de trouver un matin cette verticale flèche qui va nous élever. Pas un pouvoir, non : une puissance de pousser, pousser encore, pousser par grappes, pousser dans tous les sens comme un feu de prairie qui se répand et qui peut aussi grimper aux cimes des arbres sans jamais rien brûler, puisqu'il est fait d'eau vive et de vivant. (para. 21)

C’est par cette puissance de se mouvoir que l'eutopie se dissémine et passe d'île à continent.

La question du caractère figé et permanent de l'organisation eutopique classique est également traitée dans Les Furtifs (Damasio, 2019). Des affinités unissent les furtifs - ces êtres en perpétuelle métamorphose et hybridation - et les habitats autogérés des groupes libertaires en lutte contre la privatopie, parmi lesquelles figure l'eutopique Cité des Métaboles, construite dans les interstices de la ville "libérée " d'Orange. Comme l'affirme Lorca, la Cité est conçue pour évoluer et muter et "[1]'ensemble sonnait pluriel et baroque, métamorphique et instable » (p. 385), qualités qui siéent tout aussi bien aux furtifs, pour qui elle représente un «temple possible» (p. 385).

Les groupes libertaires voient d'ailleurs dans les furtifs une inspiration dans leur lutte contre la surveillance et pour la reprise des villes libérées ; ce sont les figures de proue des victimes de la ségrégation spatiale :

Dans [...] [leurs] discussions, on sent potentiellement un pont politique se faire entre les furtifs et [...] [la] lutte sociale. Le furtif, dans les représentations qui émergent, c'est le clandestin, l'insaisissable, le migrant intérieur. Celui qui assimile et transforme le monde. (p. 388) 
Sous l'inspiration des furtifs, les mouvances libertaires mettront d'ailleurs en place leur projet eutopique à la fin du roman, soit une première série de villes autogérées. Toutefois, les furtifs constituent davantage que le simple porte-étendard de la résistance; ils allégorisent également l'ouverture face à l'altérité et la mixité sociale en vertu de leur capacité à s'hybrider avec ce qui les entoure. Il s'agit d'une manière radicale de briser les enclaves entre soi et l'autre.

À l'ouverture et au goût pour la mixité sociale s'ajoute la préférence de l'eutopie damasienne pour le dissensus, c'est-à-dire " une dynamique, une tension dissensuelle collective forte, avec des positions qui changent sans cesse tout en maintenant des conditions de collaboration » (Damasio et Carabédian, 2016, p. 84). Ce goût pour le dissensuel s'oppose directement au principe de l'eutopie classique qu'est la réglementation (Pagès, 2000), soit le fait que l'ensemble des activités s'y voient fortement organisées et contrôlées en vue de construire une société prévisible et harmonieuse.

\subsection{La planification urbaine}

L'œuvre de Damasio remet enfin en question l'importance de la planification urbaine dans l'organisation spatiale de l'eutopie classique, qui se manifeste dans sa rationalisation extrême (Pagès, 2000) et dans son obsession cartographique, une obsession pour le monitorage en continu des flux qui la composent, soit les informations, les biens et les personnes.

Dans la nouvelle "C@PTCH@ »(Damasio, 2012), la Ville incarne de manière exacerbée la rationalisation de l'espace. Elle

se compose d'un nombre indéfini [...] de places hexagonales. De chacune de ces places partent six avenues. Chaque avenue est bordée de huit bâtiments accolés de seize mètres de large sur seize de long qui sélèvent et s'enfoncent au-dessus et en dessous du sol à une hauteur aléatoirement fixée entre deux et huit étages. (p. 85)

Sa conception se veut systématique et géométrisée à l'excès, de sorte qu'elle est inhabitée car impropre à l'habiter, intégralement « architecturé[e] et quadrillé[e] de capteurs » (p. 86), elle représente la surveillance sous une forme exacerbée, ses drones et dispositifs d'analyse biométrique y quadrillant l'intégralité de l'espace et dématérialisant les individus passant dans leurs faisceaux. Cette conception du milieu urbain relève d'une « discipline quadrillante » (Systar, 2012, p. 265) et lie la "prédominance du carré, du cube, dans la Ville, image de la "pixellisation" du monde » (p. 265), à l'idée, puisée chez Foucault (1975) selon laquelle « [p] our contrôler le réel, il faut le quadriller : le découper en carrés [...] et tisser un réseau où le pouvoir se trouve partout prolongé et démultiplié » (Systar, 2012, p. 265). Ce pouvoir est efficace « dans la mesure où l'arme inédite qu'il utilisera sera la mise en réseau généralisée des regards et des visibilités » (p. 265), mais aussi la traduction du monde sous la forme de données quantifiables et analysables. 
Dans Les Furtifs, la planification urbaine, couplée à la privatisation des espaces publics, accentue la ségrégation socio-spatiale. L'accès aux logements et aux lieux et services publics comme les parcs et les routes est désormais limité en fonction des forfaits citoyens contractés, variables selon le statut socioéconomique de chacun. Les dispositifs de surveillance contribuent à cette ségrégation en gérant les accès à la ville en fonction dudit forfait.

La rationalisation s'incarne ici dans la personnalisation et le contrôle de l'espace à des fins sécuritaires et de divertissement :

Sans trace, il n'y a pas de contrôle possible [...]. Pas de sécurité durable. Et sans trace surtout, nos algorithmes publics ne peuvent pas personnaliser votre expérience de la ville. Comment vous proposer la ville que vous méritez si l'on ne sait pas ce que vous y aimez ? Si l'on ne sait rien de vos habitudes, de vos parcours préférentiels, de vos goûts? (p. 615)

Au quadrillage de l'espace imposé par la planification urbaine, Les Furtifs oppose des pratiques habitantes mobilisées par la résistance libertaire afin de se réapproprier la ville. Parmi elles figure la stégophilie, un sport axé sur l'escalade des toitures et édifices en milieu urbain. Alors que les villes s'avèrent " trop conçues... trop vécues du sol ", les stégophiles préfèrent " trouver d'autres chemins, des trajets à nous qui ne décalquent pas les rues... des obliques, des traçantes...» (p. 220). Cette pratique se double d'un projet politique visant à habiter les interstices de la ville en dehors de toute logique marchande : ses adeptes occupent alors les toits des propriétés privées, installent yourtes et cabanes à l'aide de matériaux de récupération, recyclant la ville pour la redonner à ses habitants et opposant à la privatopie un habiter axé sur la mise en commun de l'espace et des ressources. Habiter les interstices favorise également un mouvement de déségrégation socio-spatiale, le but étant de "permettre que des gens se mélangent [...] brasse[r] des niveaux d'habitation et des populations qui ne se croiseraient normalement jamais » (p. 222). Il s'agit alors d'échapper à la logique de clubisation de la privatopie, et non uniquement à son plan urbanistique ou à ses dispositifs de surveillance.

\section{Conclusion}

Chez Damasio (2019), des pratiques habitantes dissidentes opposent le regard froid et cartésien de l'urbaniste, du gestionnaire et de toute autre incarnation du pouvoir dans le cadre d'une société surveillée et privatisée à celui de l'enfant. Les dissidents, en effet, "voient la ville comme une enfant pourrait la voir. Pour eux, un toit n'est pas la couverture d'un bâtiment qu'il sert à étanchéiser et point barre : c'est un îlot de possibles au-dessus d'une mer gris muraille » (p. 383). Or, avant d'être un programme, l'eutopie est avant tout un jeu sur les possibles latéraux à la réalité. Être mu par une aspiration eutopique revient alors à voir non ce qui est actuel, mais ce qui 
existe en puissance, comme les enfants le font tout naturellement. De ce regard " paraspectif ", Les Furtifs rend bien compte lorsque Lorca, à force de traquer les furtifs, s'hybride avec eux. Ce faisant, il fait non seulement preuve d'une ouverture radicale face à l'altérité, mais il manifeste également une capacité à ouvrir un champ des possibles et à entrevoir des alternatives afin de transformer la société. Ainsi, l'hybridité acquise lui confère la capacité d’entrevoir le monde au « conditionnel futur », à voir ce qui n'y figure qu'en germe :

[Sahar] Tu sens le monde comme eux [les furtifs] [...]. Quelque chose te parle ? [...]

[Lorca] Pas vraiment, non. J'ai juste l'impression de me parler au conditionnel [...] Je dérive parmi le possible, l'alternative [...]

[Sahar] Tu sais comment on appelle cette forme verbale en littérature ? [...]

[Lorca] Le conditionnel ?

[Sahar] L'irréel. C'est une conjugaison qui exprime une hypothèse irréaliste, irréalisable. Le latin distingue l'irréel - l'irréel du présent, l'irréel du passé - et le potentiel. Ces trois formes sont rendues en français par le conditionnel, oui, tu as raison. (p. 281)

Cette forme verbale, préférée à l'eutopie classique comme programme, c'est le langage eutopique par lequel on entrevoit les possibles latéraux à la réalité sans tomber dans l'irréel et on aspire à la création de villes autogérées et à la restauration d'un vivre-ensemble sur les ruines de la privatopie ; un habiter et un co-habiter autres et désirables. Ce langage fait d'ailleurs écho à « Hyphe...? ». L'hyphe métaphorise non seulement les liens qui unissent le collectif, mais également la perspective eutopique elle-même, car comme le rappelle le narrateur de la nouvelle, "son nom si gracieux [...] sonne comme le Si si prometteur des anglophones : "if..." (para. 21), le «si » du conditionnel futur.

\section{RÉFÉRENCES}

Aïm, O. (2020). Les théories de la surveillance. Paris : Armand Colin.

Baccolini, R. et Moylan, T. (2003). Introduction: Dystopia and Histories. Dans R. Baccolini et T. Moylan (dir.), Dark horizons: Science fiction and the dystopian imagination (p. 1-12). New York : Routledge.

Berque, A. (2000). Écoumène: Introduction à l'étude des milieux humains. Paris : Belin.

Booker, M. K. et Thomas, A.-M. (2009). The Science-Fiction Handbook. Chichester et Malden : Wiley-Blackwell.

Damasio, A. (2004). La Horde du Contrevent. Clamart : La Volte.

Damasio, A. (2012). C@PTCH@. Dans Aucun souvenir assez solide (p. 74-106). Clamart : La Volte.

Damasio, A. (2013) [1999]. La Zone du dehors. Clamart : La Volte.

Damasio, A. (2018). Hyphe...? Vice. https://www.vice.com/fr/article/bj3b7d/alain-damasioimagine-la-zad-en-2045

Damasio, A. (2019). Les Furtifs. Clamart : La Volte. 
Damasio, A. et Carabédian, A. (2016). « Utopier le désir ! » : entretien avec Alain Damasio. Tumultes, 47, 73-90.

Damasio, A. et Lévy-Provençal, M. (2016). Demain, serons-nous très humains plutôt que transhumains ? https://www.huffingtonpost.fr/alain-damasio/humanisme-transhumanis me_b_6998646.html

Deleuze, G. et Guattari F. (1980). Capitalisme et schizophrénie : mille Plateaux. Paris : Éditions de Minuit.

Duret, C. (2019). Le goût pour le Moyen Âge dans les fictions post-catastrophiques contemporaines : une lecture mésocritique. Savoirs UdeS. https://savoirs.usherbrooke.ca/handle/11143/16057

Duret, C. (2021). Le jeu vidéo Remember Me et le roman Les furtifs : deux dystopies de la surveillance dans l'œuvre d'Alain Damasio. Intercâmbio, 13, 68-91.

Engélibert, J.-P. (2015). Après la catastrophe, l'utopie. Dans J.-P. Engélibert et R. Guidée (dir.), Utopie et catastrophe : revers et renaissances de l'utopie (XVI ${ }^{e}-X X I^{e}$ siècles) (p. 239-253). Rennes : Presses universitaires de Rennes.

Faye, É. (1993). Dans les laboratoires du pire. Paris : Corti.

Foucault, M. (1975). Surveiller et punir. Paris : Gallimard.

Fougier, E. (2016). Les Zadistes : un nouvel anticapitalisme. Paris : Fondapol.

Fries-Paiola, C. et De Gasperin, A. (2014). Introduction : les pratiques habitantes au cœur de la recherche contemporaine sur les «lieux de la ville ». Revue Géographique de l'Est, 54(3-4), 1-14.

Funcke, H.-G. (1988). L'évolution sémantique de la notion d'utopie en français. Dans H. Hudde et P. Kuon (dir.), De l'utopie à l'Uchronie : formes, significations, fonctions (p. 18-37). Tübingen : Gunter Narr Verlag.

Hardt, M. et Negri, A. (2000). Empire. Cambridge : Harvard University Press.

Jenkins, H. (2006). Convergence Culture: Where Old and New Media Collide. Londres, New York : NYU Press.

Lapouge, G. (1973). Utopie et civilisations. Paris, Genève : Weber.

Lazzarotti, O. (2006). Habiter, aperçus d'une science géographique. Cahiers de géographie du Québec, 50(139), 85-102.

Levitas, R. et Sargisson, Lucy. (2003). Utopia in Dark Times: Optimism/Pessimism and Utopia/Dystopia. Dans R. Baccolini et T. Moylan (dir.), Dark horizons: Science fiction and the dystopian imagination (p. 13-27). New York : Routledge.

McKenzie, E. (1996). Privatopia: Homeowner Associations and the Rise of Residential Private Government. New Haven : Yale University Press.

Mongin, O. (2013). La ville des flux : l'envers et l'endroit de la mondialisation urbaine. Paris : Fayard.

Moylan, T. (2000). Scraps of the Untainted Sky: Science Fiction, Utopia, Dystopia. Boulder : Westview Press.

Moylan, T. (2014). Demand the Impossible: Science Fiction and the Utopian Imagination. Berne : Peter Lang AG.

Munier, B. (2013). À l'ombre de l'utopie : la contre-utopie et la dystopie. Dans E. Letonturier (dir.), Les Utopies (p. 115-130). Paris : CNRS éditions.

Nel, N. (1999). Des dispositifs aux agencements télévisuels (1969-1983). Hermès, La Revue, $25,131-141$.

Pagès, D. (2000). Des mondes parfaits aux mondes possibles : les territoires équivoques de l'utopie. Quaderni, 41, 43-63. 
Rosselin, C. (2002). Pratiques habitantes dans des logements d'une seule pièce. Communications, 73, 95-112.

Ruyer, R. (1950). L'utopie et les utopies. Paris : Presses universitaires de France.

Sargent, L. T. (2005). Pour une défense de l'utopie. Diogène, 209, 10-17.

Séguy, J. (1971). Les sociétés imaginées : monachisme et utopie. Annales : Économies, Sociétés, Civilisations, 26(2), 328-354.

Stock, M. (2004). L'habiter comme pratique des lieux géographiques. EspacesTemps.net. https://www.espacestemps.net/articles/habiter-comme-pratique-des-lieux-geographiques

Systar. (2012). Portrait de Damasio en aérophone. Dans Aucun souvenir assez solide (p. 249270). Clamart : La Volte.

RÉSUMÉ : Cet article propose une analyse du discours consacré par le roman Les Furtifs et les nouvelles «C@PTCH@ » et «Hyphe...?? », de lécrivain de science-fiction français Alain Damasio, à la question de l'habiter ; un discours partagé entre l'eutopie (ou utopie positive) et la dystopie (ou utopie négative). Nous verrons que ces œuvres, caractéristiques de la dystopie critique, ne se contentent pas de porter une appréciation négative de la conjoncture actuelle en la matière ni de proposer une vision alternative de ce qu'habiter le monde doit - ou pourrait - signifier, mais qu'elles interrogent également les limites de l'utopie classique pensée en tant que programme, ceci afin de proposer, par le biais d'un discours méta-utopique (ou discours sur le discours utopique), cette fois, une conception de l'utopie comme aspiration et comme jeu sur les possibles latéraux à la réalité.

Mots-clés : Alain Damasio, dystopie critique, habiter, discours méta-utopique, privatopie, utopie

\section{Inhabiting the interstices and their possibilities: The utopian and meta-utopian discourses of Les Furtifs, “C@PTCH@”, and “Hyphe...?" by Alain Damasio}

ABSTRACT: This paper offers an analysis of the utopian discourse in the novel Les Furtifs and the short stories “C@PTCH@” and "Hyphe...?", all coming from the French science fiction writer Alain Damasio, from the perspective of the issue of inhabitation. We will see that the three dystopias do not content themselves with taking a critical look at the current situation or offering an alternative vision of what inhabiting the world must - or could - signify. To the contrary, they also question the limits of a classical utopia conceived as a program, in order to propose, by means of meta-utopian discourse, a concept of utopia as an aspiration and "a game on the possibilities lateral to reality".

Keywords: Alain Damasio, critical utopia, inhabitation, meta-utopian discourse, privatopia, utopia 
